\title{
Shape measurement by using basis functions
}

\author{
Guoliang $\mathbf{L i}^{1,2}$, Bo $\mathrm{Xin}^{2}$ and Wei Cui ${ }^{2}$ \\ ${ }^{1}$ Purple Mountain Observatory, Chinese Academy of Sciences, \\ 2 West Beijing Road, Nanjing 210008, China. \\ email: guoliang@pmo.ac.cn \\ ${ }^{2}$ Department of Physics, Purdue University, 525 Northwestern Ave., \\ West Lafayette, Indiana 47907
}

\begin{abstract}
Gravitational lensing is one of most promising tools to probe dark energy and dark matter in our Universe. Lensing by larger-scale structures distorts the shape of background galaxies. For ground-based observations the shape is further distorted by atmospheric turbulence and optical distortions. Many algorithms were proposed to measure the shear signal but the systematic biases are still too large to be acceptable for the larger-sky surveys in the future. I will present our new algorithms for PSF reconstruction and shape measurements based on several sets of basis functions.
\end{abstract}

Keywords. gravitational lensing, shape, model fitting, basis function.

\section{Fast calculating of model parameters}

To measure the cosmic shear, the problem in hand is to determine the intrinsic (or pre-seeing) shape of a galaxy based on its observed shape. In practice, the galaxy image is noisy, which makes deconvolution challenging. As a first step, it is necessary to accurately quantify the observed shape of galaxies based on noisy images. In this article, we present a set of efficient algorithms specifically for this task. Usually, no less than six parameters are needed to model the light distribution of an object, including the centroid position $(x, y)$, the ellipticity $\left(g_{1}, g_{2}\right)$, the normalization $I_{0}$, and the profile parameter $(\mathrm{s})$. High-dimensional parameter search is very time consuming, especially when the number of objects is large, and tends to be trapped at local minima. Instead of brute-force fitting, we propose to derive the centroid position, ellipticity, and normalization of a light distribution numerically and thus reduce the number of parameters that need to be determined through fitting. Similar effort has been previously undertaken. Miller et al. (2007) and Kitching et al. (2008) proposed a fast-fitting algorithm in Fourier space, which also takes into account the effects of point spread function (PSF). Here, we will only focus on how to reproduce the observed images of galaxies and stars respectively. Armed with these two kind of smooth images, deconvolution could be easily performed and the intrinsic shape of the galaxies could be estimated.

To test the efficiency of our algorithm, we simulate 500 galaxy images based on the Sersic model. The ellipticities are chosen randomly and Gaussian noise was added on the center position and the values of each pixel. Then the center and the ellipticity was estimated by using the above algorithms. Meanwhile we use the MINUIT minimization program (James \& Roos 1975) as the brute-force method to fit these parameters. We show the differences in centroiding and the ellipticity in Fig.1. We can see that the calculated results are a little worse than that of model fitting but they are still good enough. And we should keep in mind that: 1) the process is accelerated by a factor of magnitude, 2) the shape roughly estimated parameters is not our final goal. They could be used as the 

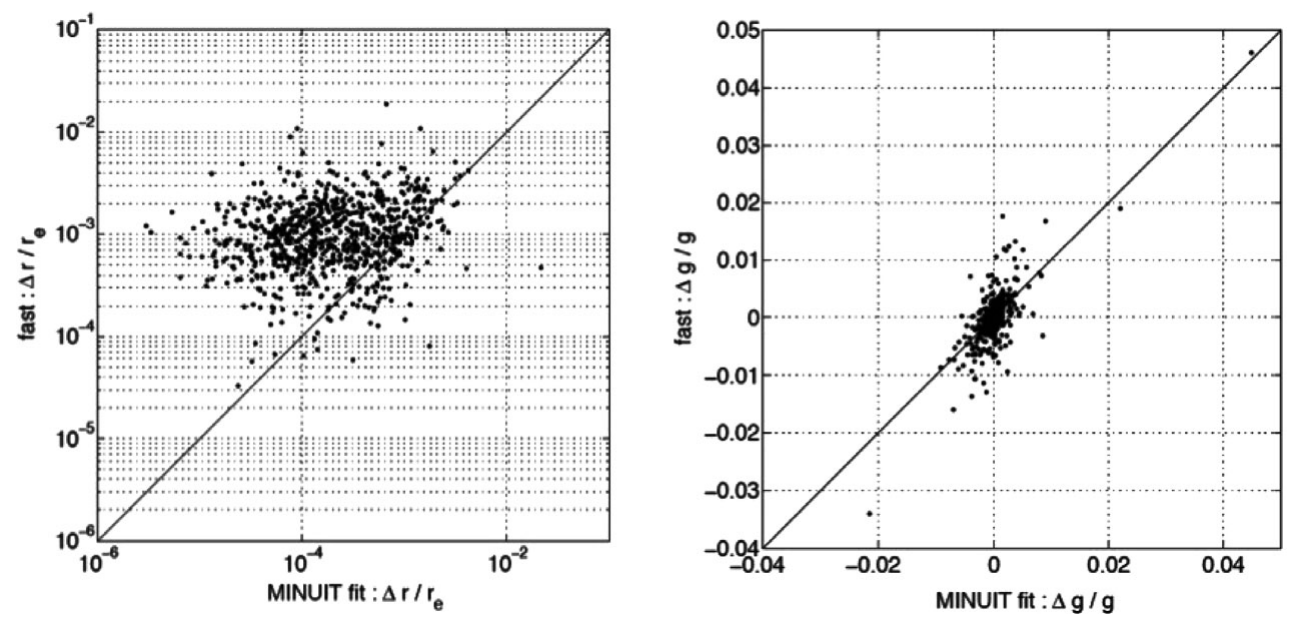

Figure 1. The differences in centroiding (L) and the ellipticity (R) which are derived by our fast calculating algorithm and by brute-force model fitting.

input parameters to create corresponding basis to improve the real image fitting further. More details can be found in Li et al. (2012)

\section{PSF reconstruction by using gaussianlets and moffatlets}

A point source will be observed as an extended image because the photons are affected by several complex physics processes. This means that the PSF can not be represented by a simple model function. It is well known that a set of complete and orthogonal basis functions could compose any shape of image. The only problem is how many basis functions we need. Therefore we construct two kinds of basis function to model the PSF and test their efficiency. For simplicity, we just consider the ellipticity of the PSF but ignore the higher-order moments.

Gaussianlets is a reduced version of shapelets (Massey \& Refregier 2005) where we only keep the basis functions with $m=0$, i.e., the basis functions are circular symmetric and contain no angular components. For a given image of star, we perform our fast calculating to derive the center and the ellipticity. Then the image is fitted by using a Gaussian function with same center and ellipticity. The resulting width is used as the only parameter to create the basis function set. All of the basis functions are reshaped into elliptical symmetry according to the measured ellipticity and linear minimizing is performed to obtain the coefficients for each basis function

Moffalets is another kind of basis function the 0th order of which is the Moffat function itself. Moffat model has been believed one of the optimal model for the PSF. Now with the high order corrections we could improve the model to an new level. Our gaussianlets and moffatlets has been tested in the star challenge of GREAT10 program and got high scores.

\section{Galaxy reconstruction by using sersiclets}

The Sersic model is successful to fit the light distribution of galaxies. But it is not always successful because of the complexity of real galaxies. Shapelets were first proposed to mimic the light distribution of any type of galaxies since they are complete and orthogonal basis functions. But shapelets are not optimal because they are not compact 


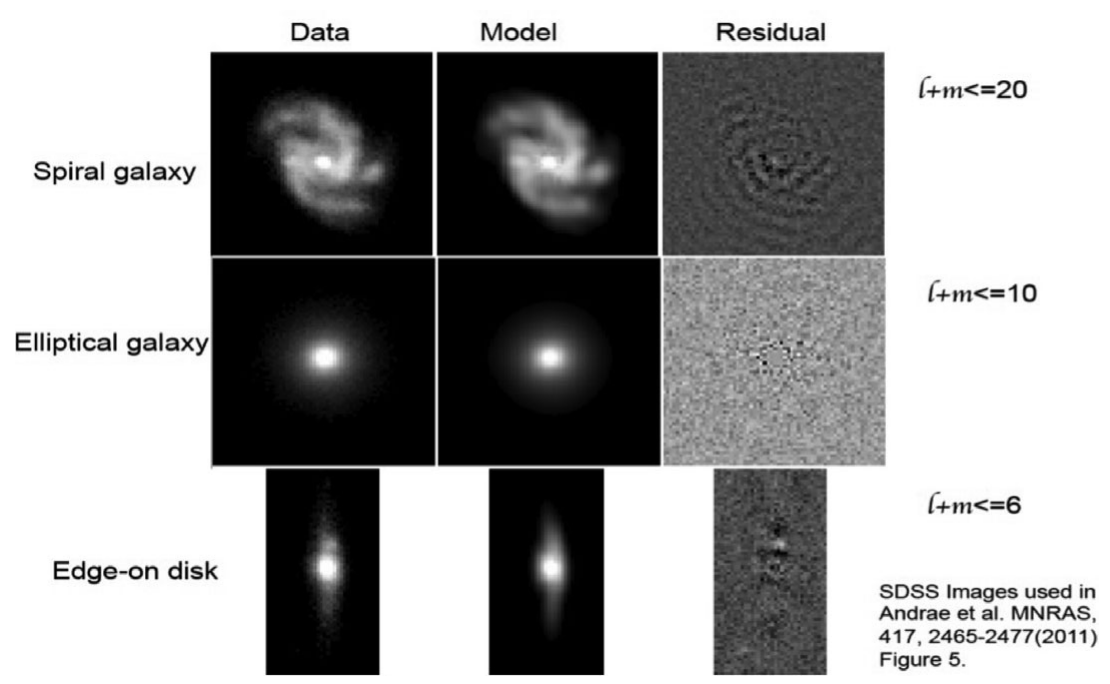

Figure 2. Image reconstruction by using elliptical sersiclets.

enough. From this point of view, Andrae et al. (2011) proposed another kind of basis function based on the Sersic model which are called Sersiclets. However either shapelets and Sersiclets need very high orders to reproduce an high ellipticity. Therefore we extend the standard sersiclets to elliptical sersiclets as same as we did for gassianlets and moffatlets and reproduced the three images which are used in their work. We show the fitting results as Fig. 2.

\section{Summary}

In this poster we present a set of algorithms to reconstruct the image of star and galaxy in a quick and accurate way. 1) For the issue of model fitting, we propose a new way to search the parameters separately. The center and ellipticity can be derived by calculations and our algorithm thus decrease the fitting dimension dramatically. 2) Gaussian and Moffat models are usually used to represent the PSF but they are not perfect because the PSF is too complex to be described by just one or two parameters. We proposed two kinds of basis function named gaussianlets and moffatlets to implement high-order correction, so that we could reproduce any kind of PSF accurately in principle. 3) We also improved the reconstruction of galaxy images by using elliptical sersiclets. They are able to reproduce the light distribution of any type of galaxy with higher efficiency than what shapelets can achieve.

\section{References}

Andrae, R., Melchior, P., \& Jahnke, K. 2011, MNRAS, 417, 2465

Kitching, T. D., Miller, L., Heymans, C. E., van Waerbeke, L., \& Heavens, A. F. 2008, MNRAS, 390,149

James, F. \& Roos, M. 1975, Comput. Phys. Commun. 10, 343

Li, G. L., Xin, B., \& Cui, W. 2012, arXiv:1203.0751

Massey, R. \& Refregier, A. 2005, MNRAS, 363, 197

Miller, L., Kitching, T. D., Heymans, C., Heavens, A. F., \& van Waerbeke, L. 2007, MNRAS, 382,315 